\title{
Interaction Grammars
}

\author{
Guy Perrier \\ LORIA, Université Nancy2 \\ BP 239 \\ 54506 Vandœuvre-lès-Nancy Cedex France \\ e-mail: perricr@loria.fr
}

\begin{abstract}
Interaction Grammars (IG) are a new linguistic formalism which is based on descriptions of underspecified trees in the framework of intuitionistic lincar logic (ILL). Syntactic composition, which is $\mathrm{cx}$ pressed by deduction in lincar logic, is controlled by a system of polarized fcatures. In this way, parsing amounts to generating models of tree descriptions and it is implemented as a constraint satisfaction problem.
\end{abstract}

\section{Introduction}

IG can be presented as an attempt to bring together fundamental ideas, some coming from Tree Adjoining Grammars (TAG) and others from Categorial Grammars (CG), in order to overcome the specific limitations of each of these formalisms. The computational and linguistic relevance of TAG lies in its adjunction operation (Joshi et al., 1975; Kroch and Joshi, 1985) but the simplicity of its mechanism has a counterpart in the inflation of the lexicons that are required for expressing all grammatical phenomena of a language. Every time that a word is used in a new syntactic context, which can differ only by word order for example, a new elementary tree, which encodes this context, must be added to the lexicon in a direct manner or by means of a lexical rule. In this way, lexicons quickly become colossal, very awkward to use and very hard to maintain.

Recent works aim to solve this problem by factorizing linguistic information with the notions of underspecified trees and tree descriptions. These notions were introduced for TAG by Vijay-Shanker with the motivation of making adjunction monotone (VijayShanker, 1992). Now, they are cxploited fruitfully in various directions: for structuring TAG lexicons in hierarchical systems of modules (Candito, 1999) or for cxpressing semantic ambiguity (Muskens and Krahmer, 1998; Egg et al., 1998) for instance. At the same time, these notions are a way of relaxing the primitive adjunction operation in order to capture linguistic phenomena: such a possibility is exploited by (Rambow et al., 1995) within D-Tree Grammars and by (Kallmeycr, 1999) within Tree Description
Grammars.

Unfortunately, the counterpart of a more flexible framework is often over-gencration and a loss of computational efficiency in the absence of principles for controlling syntactic composition. By looking at $C G$, we can find some answers to this preoccupation: a fundamental idea of $\mathrm{CG}$ is that grammatical constituents are viewed as consumable resources (Retoré, 2000); these resources are divided into positive and negative resources which are complementary and scarch to noutralize themselves mutually. The core of CG is the Lambck Calculus (Lambek, 1958): by combining resource sensitivity and order sensitivity, this logic is a good candidate for representing the syntax of natural languages but at the same time, this combination entails a rigidity which limits its expressive power greatly. An appropriato way of relaxing this rigidity constitutes an important rescarch area in CG (Moortgart, 1996).

The principle of IG is to combine the powerful notion of under-specified trec description linked to the TAG philosophy with control of syntactic composition by a system of polarities in accordance with the CG philosophy. More precisely, the basic objects of IG are syntactic descriptions which express dependencies between syntactic constituents in the shape of under-specificd trees. Word order is reforred to the same level as morphological information, which is represented by a system of features which are linked to the nodes of syntactic descriptions. Whereas a feature is usually a pair (attribute, value), an IG feature is a triplet (attribute, polarity, value) where polarity can take one of the three values $-1,0$ or +1 and bchaves like an electrostatic charge: for instance, a noun phrase which is waiting to receive a syntactic function in a sentence, carries a negative feature of type funct while a finite verb which is searching for its subject, carries a positive feature funct with value sujj. Attraction between these dual features will make possible the fact that the verb finds its subject and, simultancously, the noun phrase finds its function in the sentence. (Muskens and Krahmer, 1998) also recognized the necessity of introducing the notion of polarity in tree descrip- 
tions as a mochanism for controlling syntactic composition; the difference with respect to IG lies in the gramularity of the polarization which is finer in JG: in their proposal, the polarized objects are constituents, that is description nodes, whereas in IG one constituent can include several features with opposite polaritics.

The framework which is chosen for representing syntactic descriptions in this paper is that of linear logic (Girard, 1987), more precisely a fragment of ILL (Lincoln, 1992). The resource sensitivity of linear logic allows one to express the fact that polarized features behave as consumable resources in IG: a positive fcature has to find its dual feature once and only once. If we try to use classical or intuitionistic logic for modelling IG, the contraction and weakening rules, which are inherent in these logies, ontail a loss of resource-sensitivity: for instance, a verb could tako two subjects by appealing to the contaxaction rule and some noun phrases would not need to find their syntactic role in a sentence by appealing to the weakening rule. By discarding these two rules, linear logic provides a framowork that exactly corresponds to the "olectrostatic" laws that control polarized features.

In this framework, parsing takes the shape of logical deduction of closed syntactic descriptions from any syntactic descriptions: a description is said to be closed when it represents a completely specified syntactic tree where all features aro noutralized.

If lincar logic provides an elegant framework for representing $I G$, it gives no method for parsing efficiently and avoiding the combinatory explosion that; may follow from the flexibility of the formalism. An answer to this problem is given by the paradigm of constraint solving. Parsing a phrase can be regarded as generating models of the partial description which is provided by a lexicon for the words of this phrase. The process is monotone and can be expressed as a constraint satisfaction problem. This constraintbased approach was inspired by the work of (Juchier and C., 1999; Duchicr and Thater, 1999) on dominance constraints. (Blache, 1999) shows the advantages of such an approach both from a linguistic and computational viewpoint with the formalism that he proposes and he calls Property Grammars.

\section{Syntactic descriptions as linear logic formulas}

IG are formally defined as an ILL theory. Basic objects are syntactic descriptions which are represented by lincar logic formulas in the following form: Descr $:=$ Domin $\mid$ Feat $\mid$ Descr $\otimes$ Descr $\mid$ Descr \& Descr

If a syntactic description concerns the dominance relation between syntactic constituents, it has the type Domin; if it concerns the features which are used for characterizing syntactic or semantic properties of constituents, it has the type Feat. Finally, a description can be built recursively from two descriptions in two ways, which are expressed by the two linear logic conjunctions: the multiplicative tensor $(\Theta)$ and the additive with (\&).

\subsection{Multiplicative and additive conjunction of resources in descriptions}

$A$ description $D_{1} \otimes D_{2}$ requires all resources of both descriptions $D_{1}$ and $D_{2}$ while a description $D_{1} \& D_{2}$ requires either the resources of $D_{1}$ or the resources of $D_{2}$ but not both. This use of the two linear logic conjunctions is consistent, with their left introduction rules in the linear sequent calculus:

$$
\frac{F_{1}, F_{2}, \Gamma \vdash G}{F_{1} \otimes F_{2}, \Gamma \vdash G} \otimes_{L} \frac{F_{1}, \Gamma \vdash G}{F_{1} \& F_{2}, \Gamma \vdash G} \&_{L 1} \frac{F_{2}, \Gamma \vdash G}{F_{1} \& F_{2}, \Gamma \vdash G} \&_{L 2}
$$

In this way, it is possible to describe all syntactic configurations of a word with a single lexical entry under the form of a syntactic description: common parts of these configurations are factorized whercas specific parts are distributed into altermations linked together with the connective with. For instance, a possible lexical entry for the finite verb voit in French has the shape $D_{v o i t}=D_{1} \otimes\left(D_{2} \& D 3\right) \otimes\left(D_{4} \& D 5\right)$ : $D_{1}$ contains information related to the subject which is common to all uses of the verb voit; $D_{2}$ expresses the canonical order subject-verb in the sentence that is headed by the verb voit whereas $D_{3}$ expresses the reverse order for which the subject must be realized under some conditions, such as in the phraso Marie que voit Jean; $D_{4}$ expresses that the verb has an explicit object whoreas $D_{5}$ corresponds to circumstances where this object is not present, such as in the sontence Jean voit.

\subsection{Under-specification of dominance between constituents}

$A$ description of type Domin has the following form: Domin $::=\tilde{N}$ ode $>[$ LNodes $] \mid$ Node $>$ Node $\mid$ Node $>^{*}$ Node

LNodes ::= $\epsilon \mid$ Node LNodes

A predicate $N>\left[N_{1}, \ldots, N_{p}\right]$ states that the constituent $N$ is decomposed into the subconstituents $N_{1}, \ldots, N_{p}$. The order between these sub-constituents is only used for identifying each one without any linguistic meaning; word order is dealt with at the same level as morphological information by means of features.

A predicate $N_{1}>N_{2}$ expresses that $N_{2}$ is an immediate sub-constituent of $N_{1}$. Such a predicatc is used when only partial information on the sub-constituents of a phrase is available.

A predicate $N_{1}>^{*} N_{2}$ expresses that $N_{2}$ is embedded in $N_{1}$ at an undetermined depth. For instance, if we continue with description $D_{1}$ related to the verb voit, we can assume that it contains the formula 
$\left(N_{3}>\left[N_{4}, N_{5}\right]\right) \otimes\left(N_{4}>^{*} N_{6}\right)$ which is interpreted as follows: the verb phrase $N_{3}$ is constituted of the verb $N_{4}$ and its object $N_{5} ; N_{6}$ represents the bare verb whercas $N_{4}$ represents the verb which has been possibly modified by a clitic, a negation or an adverb. Under-specification of the dominance relation $N_{4}>^{*} N_{6}$ leaves all these modifications open. Under-specification of dominance between constituents goes beyond TAG adjunction in that the nodes which are in a dominance relation do not necessarily have the same grammatical category and thus linguistic phenomena like wh-extraction can be expressed easily in this way.

\subsection{Polarized and under-specified features}

Descriptions of type Feat, related to features, have the following form:

$$
\begin{aligned}
& \text { Feat }::=\text { Node }: \text { Attr Pol Val } \mid \\
& \text { Var } \in \text { Dom } \mid \text { Var } \notin \text { Dom } \\
& \text { Pol } \quad::= \leftarrow|=| \rightarrow \\
& \text { Val } \quad::=\text { Const } \mid \text { Var. }
\end{aligned}
$$

A feature Node: Attr Pol Val is a triplet composed of an attribute Attr, a polarity $P o l$ and a value $\mathrm{Val}$ associated with a syntactic node Node. Usually, a feature is defined as a pair (attribute, value). In $I G$, we add a polarity to this pair so that fcatures behave like clectrostatic charges: a positive feature Attr $\rightarrow$ Val seeks a negative feature Attr $\leftarrow$ Val to neutralize it and conversely while a neutral feature $A t t r=V a l$ only acts as a filter through constraints on its value $\mathrm{Val}$ when it meets another feature of type Attr at the same node.

In all cases, $V a l$ is either a constant which is sclected from an infinite countable set $\mathcal{C}$ onst of feature values or a variable which is selected from an infinite countable set $\mathcal{V}$ ar of feature variables; then, its definition domain can be constrained by two kinds of predicates: Val $\in$ Dom and Val $\notin D o m ; D o m$ is a finite set of elements taken from Const.

Let us illustrate this presentation with a possible lexical entry for the proper noun Jean:

$$
\begin{aligned}
& D_{\text {Jeun }}=(N>[]) \otimes \\
& (N: \text { cat }=n p) \otimes\left(N: \text { funct } \leftarrow V_{1}\right) \\
& (N: \text { ord } \rightarrow 1) \otimes\left(N: \text { phon }={ }^{\prime} \text { Jean' }\right) \otimes \\
& (N: g e n=m) \otimes(N: n u m=s g) \otimes \\
& (N: \text { pers }=3)
\end{aligned}
$$

Some features are neutral by nature like agreement features: $g c n=m$ (gender $=$ male), $n u m=s g$ (number=singular), pers=3 (person=3). Others are polarized by nature too: for instance, features of type funct which express syntactic functions. In the example above, the feature of type funct is negative because the noun phrase represented by $N$ is waiting to receive a syntactic function (subject, object...); this function is not determined yet and thus it is represented by a variable $V_{1}$.

The phonological form of a constituent, is determined by a system of two fcatures: phon which gives the effective phonological form of the constituent and ord which gives the order in which its immodiate sub-constituents must be concatened to build this phonological form. For instance, we find the formula $\left(N_{1}:\right.$ ord $\left.\rightarrow V_{2}\right) \otimes\left(V_{2} \in\{12,21\}\right)$ in the description $D_{\text {voit }}$ to express that the clause which has the verb voit as its head and is represented by node $N_{1}$ is a concatenation subject-verb phrase $\left(V_{2}=12\right)$ or verb phrase-subject $\left(V_{2}=21\right)$. When a node has no children, two cases occur: the node has an empty phonological form and the value of the feature ord is 0 or the node is a lexical anchor and the value of the feature ord is 1 . In this case, the feature phon is used for retrieving the effective phonological form, which can be verified in the description $D_{J e a n}$. Polarization of phonological forms expresses that some constituents are capable of giving a phonological form while others are waiting for onc. As the previous examples shows, this polarity is not carried by the feature phon but by the feature ord. The interest, of giving privilege to the feature ord with respect to the feature phon, is twofold: we can detcrmine its value for a given node without being aware of the phonological form of the children, the effective phonological form will be rebuilt step by step from the leaves to the root of the final syntactic tree as soon as possible; another interest is that features of type ord can be dealt with like all other features; in particular, we can apply to them the same type of constraints.

Finally, it is interesting to mention that value sharing by different features is represented in an easy way by using a unique variable for the values of the concerned features.

\section{Syntactic composition as deduction in a linear theory}

By choosing a logical framework for a formal definition of IG, we find a natural way of expressing syntactic composition by means of deduction in linear logic according to the paradigm "parsing as deduction" of CG (for a broad survey of CG sec (Retoré, 2000)). An interaction grammar is lexicalized in the sense that all linguistic resources are stored in a lexicon and these resources will be combined by using inference rules of the ILL deductive system for building the acceptable sentences of the corresponding language. Since syntactic descriptions use only a fragment of this logic and if we choose the framework of the sequent calculus, only seven ILL rules are useful:

$$
\begin{aligned}
& \overline{F_{1}, \ldots, F_{n} \vdash F_{1} \otimes \cdots \otimes F_{n}} i d \\
& \frac{\Gamma \vdash G}{1, \Gamma \vdash G} 1_{I}, \frac{F_{1}, F_{2}, \Gamma \vdash G}{F_{1} \otimes F_{2}, \Gamma \vdash G} \otimes_{I} \\
& \frac{F_{1}, \Gamma \vdash G}{F_{1} \& F_{2}, \Gamma \vdash G} \&_{L 1} \frac{F_{2}, \Gamma \vdash G}{F_{1} \& F_{2}, \Gamma \vdash G} \&_{L 2} \\
& \frac{F[t / X], \Gamma \vdash G}{\forall X F, \Gamma \vdash G} \forall_{L} \frac{\Gamma_{1} \vdash F F, \Gamma_{2} \vdash G}{\Gamma_{1}, \Gamma_{2} \vdash G} c u t
\end{aligned}
$$


With respect to the usual presentation of the ILL scquent calculus (Lincoln, 1992), axiom $i d$ is defined a bit differently but this definition is equivalent to the original one for the logical fragment used by IG. Rule $V_{I}$ is a first order rule which is used here for instantiating a node variable with a concrete node or a feature variable with a concrete feature value. Beside these general rules, we need proper axioms to express properties related to dominance relations, feature polarities, foature values and phonological forms. Concerning dominance relations, we have the following proper axiom schemes:

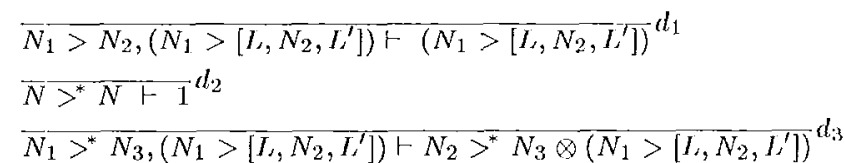
Axiom scheme $d_{1}$ expresses that immediate dominance is realized by a parent-children relation whereas axiom schemes $d_{2}$ and $d_{3}$ express that dominance is realized by finite sequences of parentchildren relations ( $L$ and $L$ ' represent sequences of node variables).

The behaviour of polarities is represented by the following propor axiom schomes:

$\overline{\left(N: A I^{\prime} V\right),(N: A=V) \leftarrow\left(N: A I^{\prime} V\right)^{p_{1}}}$

$\overline{(N: A<-V),(N: A \rightarrow V)}{ }^{-}\left(\overline{N: A \equiv V)}{ }^{p_{2}}\right.$

Propertics related to feature domains and values are expressed by the following axiom schemes:

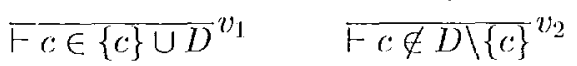

In both axiom schemes, $D$ represents a concrete finite set of feature values taken from Const and $U$ and \represent the nsual operations of union and difference of sets.

Finally, three axiom schemes are used for deducing the effective phonological form of a constituent from the order of the plionological forms of its children:

$$
\begin{aligned}
& \bar{N} \overline{>[],(N: \text { ord }=0) \vdash N>[] \Phi\left(N: \text { phon }="{ }^{\prime}\right)} p h_{1} \\
& \bar{N} \overline{>[],(N: \text { ord }=1) \vdash N>[]} p / h_{2} \\
& \overline{\left(N>\left[N_{1}, \ldots, N_{p}\right]\right.} \overline{, O, P_{1} \vdash\left(N>\left[N_{1}, \ldots, N_{p}\right]\right) \otimes P_{2}} p h_{3}
\end{aligned}
$$

Schemes $p h_{1}$ and $p h_{2}$ respectively comespond to empty categories and lexical anchors.

In scheme $p h_{3}, O$ is an abbreviation for $(N$ : ord $=c(\sigma)) ; \sigma$ is a pormutation on $[1, p]$ which expresses an order for concatenating the phonological forms $v_{1}, \ldots, v_{p}$ of the children nodes $N_{\mathrm{I}}, \ldots, N_{p}$ of $N$ and $c(\sigma)$ is a bijective encoding of this permutation with an integer. $P_{1}$ is an abbreviation for $\left(N_{1}:\right.$ phon $\left.=v_{1}\right), \ldots,\left(N_{p}:\right.$ phon $\left.=v_{p}\right)$ and $P_{2}$ an abbreviation for the product $\left(N:\right.$ phon $\left.=v_{\sigma(1)} \ldots v_{\sigma(p)}\right) \otimes\left(N_{1}:\right.$ phon $=$ $\left.v_{1}\right) \otimes \cdots \otimes\left(N_{p}:\right.$ phon $\left.=v_{p}\right)$.

A particular interaction grammar $G$ is defined by its vocabulary $\mathcal{V}_{0} c_{G}$ and by a lexicon $\mathcal{L e x}_{G}$; the vocabulary $\nu_{0} c_{G}$ includes the words used for building the language $\mathcal{L}_{G}$ gencrated by this grammar and the lexicon $\mathcal{L} e x_{G}$ associates a syntactic description to each word of $\mathcal{V} \mathrm{c}_{G}$. Now, we have to combine the resources provided by $\mathcal{L} e x_{G}$ by means of the inference rules and proper axioms of the lincar theory $\mathcal{T}$ which has just been defined to compose well-formed and complete syntactic structures of $G$ under the shape of closed syntactic descriptions. As a preliminary, we have to give a precise definition of a closed syntactic description:

A closed syntactic description is a particular syntactic description in the shape $S \otimes \Gamma$ where $S$ and $F$, respectively, represent the structural and feature parts of the description with the following conditions:

1. $\mathrm{S}$ is a product of predicates in the form $\left(n>\left[n_{1}, \ldots, n_{p}\right]\right)$, where $n, n_{1}, \ldots$, $n_{p}$ represent concrete syntactic nodes, and the structure defined by all these parent-children relations is a tree;

2. $\mathrm{F}$ is a product of predicates in the form $(n:$ attr $=v)$, where $n$, attr and $v$ represent concrete atoms, and for each pair $(n, a t t r)$ present in $\mathrm{F}$, there is exactly one feature $(n:$ att $r=v)$ in $F$.

3. For every syntactic node $n$ in $\mathrm{S}$, there is a fcature $(n:$ plon $=v)$ in $\mathrm{F}$.

Condition 1 guarantees that a closed syntactic description represents a completely specified tree. Condition 2 guarantees coherence and nentrality of the feature system which is attached at oach syntactic: node. Condition 3 guarantees the phonological well-formedness of the whole syntactic structure.

Now, let us explain how $G$ generates closed syntactic descriptions from $n$ lexical entries $D_{w_{1}}, \ldots, D_{w_{n}}$ corresponding to $n$ words $w_{1}, \ldots, w_{n}$ taken from $\operatorname{Voc}_{G}$. For this, we need an additional description $D_{\text {root to }}$ represent the root of the final syntactic tree which has the form: $\left(N_{0}>^{*} N_{1}\right) \otimes \cdots \otimes\left(N_{0}>^{*}\right.$ $\left.N_{p}\right) \otimes\left(N_{0}\right.$ : ord $\left.\leftarrow V_{0}\right)$. Node $N_{0}$ represents the root of the syntactic tree and $N_{1}, \ldots, N_{p}$ are the nodes present in descriptions $D_{w_{1}}, \ldots, D_{w_{n}}$. Then:

\section{A closed syntactic description $D$ is said to be generated from the words $w_{1}, \ldots, w_{n}$ by grammar $G$ if the sequent $\forall \vec{N} \forall \vec{V}$ (D root $\theta$ $\left.D_{u_{1}} \otimes \cdots \otimes D_{w_{n}}\right)+D$ is provable in the theory $\mathcal{T}(\vec{N}$ and $\vec{V}$ represent all node vari- ables and feature variables that are free in $\left.D_{\text {root }}, D_{w_{1}}, \ldots, D_{w_{n}}\right)$.}

$D$ describes a trec which represents the syntax of a phrase given by the feature phon of its root. If we add the prodicate $\left(N_{0}: p h o n=w_{1} \ldots w_{n}\right)$ to $D_{\text {root }}$, we transform the gencration of closed syntactic descriptions into parsing of the phrase $w_{1} \ldots w_{n}$. 
By continuing with the verb voit, let us give a very simple illustration of this mechanism. We assume that a lexicon provides us with three descriptions $D_{\text {voit }}, D_{i l}$ and $D_{\text {Jean }}$ which respectively correspond to the finite verb voit, the personal pronoun $i l$ and the proper noun Jean. As it was described in subsection $1.1, D_{\text {voit }}$ has the shape $D_{1} \otimes\left(D_{2} \& D_{3}\right) \otimes$ $\left(D_{4} \& D_{5}\right)$ and it is schematized by the following diagram:

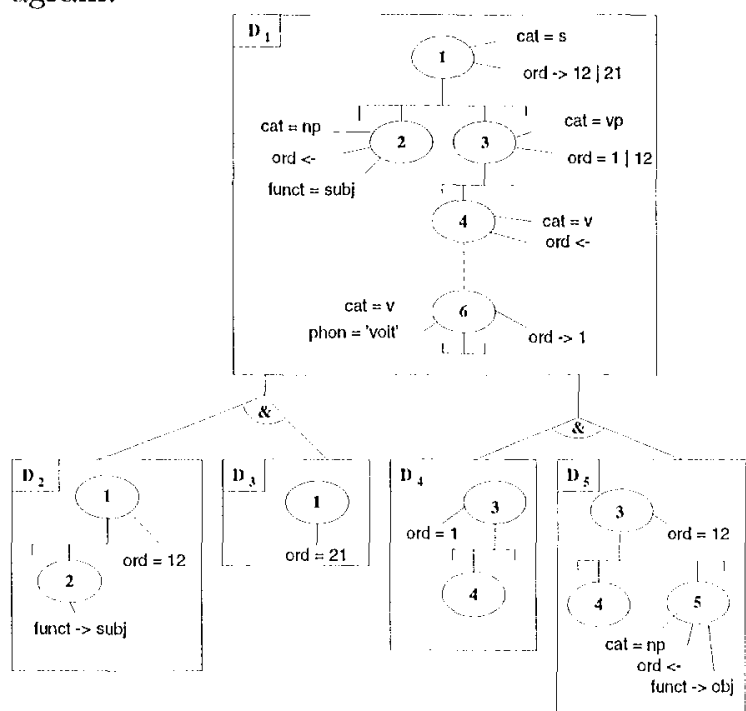

To remain readable, the diagram includes only the most significant features of every node. The notation ord $\rightarrow 12 \mid 21$ is an abbreviation for ord $\rightarrow V$ with $V \in\{12,21\}$ and ord $\leftarrow$ means that the value of the feature ord is undetermined.

Description $D_{i l}$ has a structure that is similar to $D_{\text {voit }}$ :

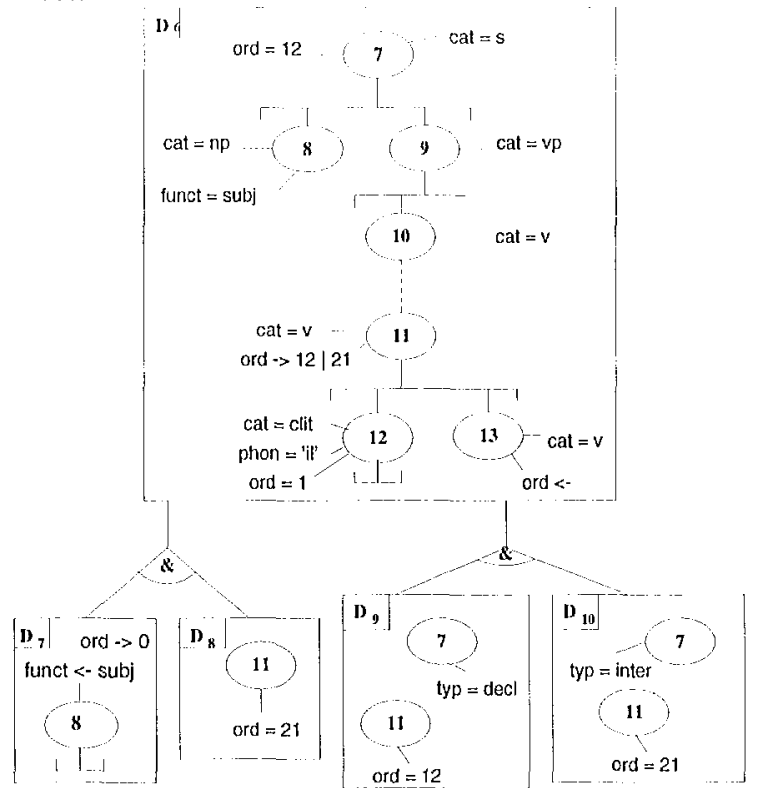

The first additive component of description $D_{i l}$, $D_{7} \& D_{8}$ represents a choice between the absence of an explicit subject in the sentence beside the per- sonal pronoun $i l$ such as in the sentence $i l$ voit Jean and the presence of this subject such as in the sentence Jean voit-il ?. The second alternative cntails that the sentence is interrogative if we ignore topicalization, which explains description $D_{8}$.

The second additive component of description $D_{i l}$, $D_{9} \& D_{10}$, represents a choice between the declarative type and the interrogative type of the sentence which depends on the relative order between the verb and the clitic.

Description $D_{J e a n}$ is reduced to the following single node:

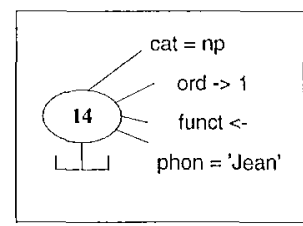

From the description $\forall \vec{N} \forall \vec{V}\left(D_{\text {root }} \otimes D_{\text {voit }} \otimes\right.$ $\left.D_{\text {Jean }} \otimes D_{i l}\right)$, it is possible to deduce three closed syntactic descriptions $D_{a}, D_{b}$ and $D_{c}$, which respectively represent the syntax of the grammatical sentences :il voit Jean, voit-il Jean? and Jean voit-il?. In concrete terms, the deduction process that leads to these three solutions consists in plugging nodes of the initial descriptions with the aim of neutralizing all polarized features while respecting dominance and feature constrains. Let us detail the resulting description $D_{b}$ by means of the syntactic tree it specifies:

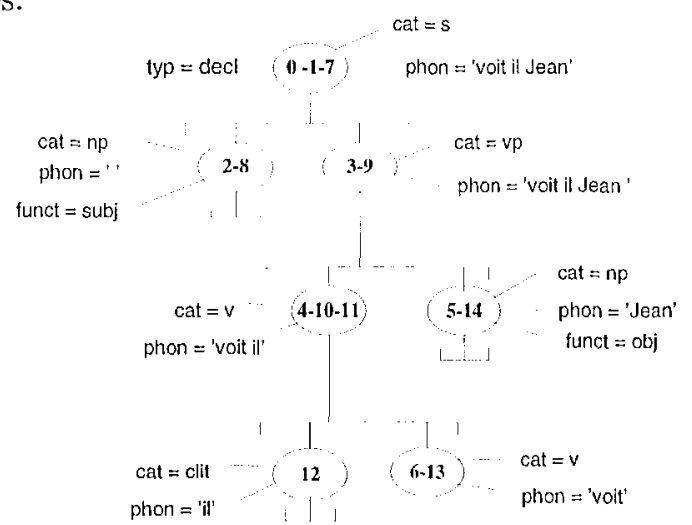

The closed syntactic description that specifies the tree above represents the syntactic structure of the sentence voit-il Jean?. The numbers that label its nodes are the traces of the nodes of the descriptions that have been plugged in the parsing process.

\section{A constraint-based implementation}

From the viewpoint of a computer scientist, a linguistic model has to show not only expressive power but also computational tractability. In the previous section, we have shown that IG computations reduce to ILL proofs. For the logical fragment that we consider here, three logical rules are a source of non- 
doterminism in proof search: $\&_{L 1}, \&_{L 2}$ and $\forall_{L}$. This takes the shape of three kinds of choice points in the parsing process: selecting the pertinent branch for every additive conjunction, identifying some nodo variables and instantiating feature variables in an a)propriate manner. The NP-completeness of the implicative fragmont of ILL (Kanovich, 1992) shows that it is hopeless to find a general parsing algorithm for IG that works in polynomial time in the worst cases. Experience has shown that, fortunately, these worst cascs rarely occur in parsing natural languages. Nevertheless, the flexibility of IG entails a combinatory explosion of the parsing process if we use a "generate and test" method and leads us to choose a more appropriate method. The specification of our problem prompts us in a natural way to a constraint-based approach as it was suggested by some proposals for similar problems (Duchier and C., 1999; Duchier and Thater, 1999).

The problem can be formulated as follows:

Given a syntactic description $D_{0}$, find all closed syntactic descriptions $D$ such that $\forall \vec{N} \forall \vec{V} D_{0} \vdash D$ is provable in the theory $\mathcal{T}$ $(\vec{N}$ and $\vec{V}$ respectively represent the node variables $N_{1}, \ldots, N_{n}$ and the features vartables $V_{1}, \ldots, V_{m}$ of $\left.D_{0}\right)$.

A fundamental property of the deduction process that leads to a solution is monotonicity so that the problem can be cxpressed as a construint satisfaction problem (CSP). A CSP is spocified from a set of variables to which constraints are applied. Herc, we consider three sets of variables, which correspond to the three kinds of choice points in the parsing procoss:

1. the set $\left\{N_{1}, \ldots, N_{n}\right\}$ of syntactic node variables;

2. the set $\left\{V_{1}, \ldots, V_{m}\right\}$ of feature variables;

3. the set $\left\{S_{1}, \ldots, S_{p}\right\}$ of selection variables; every selection variable $S_{i}$ is an integer variable which is associated with a connective \& of $D_{0}$ and which is used for indicating the rank of the component of the correspondent additive conjunction that is selected in the deduction.

Selection and feature variables are considered as finite domain variables, which imply that all feature values are encoded as integers (one exception is that features of type phon remain strings).

Node variables are encoded indirectly via finite set variables by using the method proposed in (Duchier and C., 1999). Every node variable $N_{i}$ is associated with five finite set variables $e q(i), u p(i), \operatorname{down}(i)$, side(i) and alt(i) which are used for locating the node $i$ with respect to the others in the system of dominance rolations. Because of the presence of additive conjunctions, a node $i$ which is present in the description $D_{0}$ may be absent from a solution. In this case, $e q(i)=\{i\}, \operatorname{alt}(i)=$ $\llbracket 1, n \rrbracket \backslash\{i\}, u p(i)=\operatorname{down}(i)=\operatorname{side}(i)=\emptyset$; in the case that $i$ is present in a solution, alt $(i)$ represents the nodes that are not selected in the solution whereas the selected nodes are distributed into the four sots $e q(i), u p(i)$, down $(i)$ and side $(i)$ according to their relative position with respect to $i$.

Constraints on the variables of the problem are divided into two parts:

- general constraints guarantee that the solutions $D$ are effectivo closed syntactic descriptions;

- specific constraints guarantee that the solutions $D$ are models of the initial description $D_{0}$.

\subsection{General constraints}

Treeness constraints For every node $i$, the partition of $\llbracket 1, n \rrbracket$ between $e q(i), u p(i)$, down $(i), \operatorname{side}(i)$ and alt $(i)$ guarantees that the solution is a directed acyclic graph (DAG).

For expressing that all dominance relations which structure a solution must only be realized by parcntchildren relations, we must introduce constraints in which variables of type eq(i) and selection variables appear for expressing that every selected node variable must be iclentified with a node variable which is the parent in a selected parent-children relation. In order to express that a solution is more than a DAG, that is a tree, we must add the following constraint: for every selected parent-children relation, the sets down $(j)$ for the children $j$ present in this relation must be disjoint. Such a condition can be dropped if wo want to extend the formalism to take into account resource sharing like coordination for instance; in this case, syntactic structures are no longer trees but DAGs.

Neutrality constraints Feature neutrality of a solution is guaranted by constraints which also appeal to variables of type $e q(i)$ and selection variables: for each attribute Attr, we consider two sets of sets in the shape eq $(i)$ : the first corresponds to all selected predicates in the form $(N i: A t t r \leftarrow-V)$ and the second to all selected predicates in the form $(N i: A t r \rightarrow V)$. The clcments of each of these sets must be disjoint sets and every element of the first set must be identified with one clement of the second and conversely.

Other general constraints related to foatures and phonological forms are trivial.

\subsection{Specific constraints}

Such constraints are determined by $D_{0}$. Dominance constraints are casily implemented by combining selection variables and variables of type eq(i), up $(i)$, down $(i)$, side $(i)$ (Duchier and Thater, 1999). 
Feature constraints concern both feature variables and selection variables which are all finite domain variables so that their implementation appeals to classical tools in the domain of constraint programming.

\subsection{A prototype parser for French}

We have implemented a prototype parser for French. It is written in the language $O z$ (Smolka, 1995) which combines various aspocts and modules, including constraint programming. Though the linguistic coverage of the lexicon is still limited, we have learnt lessons from the first experiments: in particular, neutrality constraints play a central role for restricting the search space, which confirms the importance of polarities for the computational efficiency.

\section{Conclusion}

Starting from TAG and $\mathrm{CG}$, we have presented a linguistic formalism which aims at better capturing the flexibility of natural language by using two notions as its basis: underspecification and polarities. In some sense, they correspond to two important propertics of natural language: ambiguity and resource sensitivity.

To regard parsing as a constraint satisfaction problem fits in with the flexibility of the formalism in terms of computational efficiency but, at the same time, it allows to go towards robustness beyond a traditional viow of parsing in which only grammatical and completely specified structures are taken into account.

The success of IG does not essentially depend on the formal properties that are usually exhibited for grammatical formalisms: the characterization of the class of languages that are generated by these grammars or the complexity of general parsing algorithms. Formal properties matter but with respect to an essential goal: to cxtend the linguistic coverage of IG from toy lexicons to massive lexical databases. For this, IG have some advantages by making it casily to factorize and modularize information: such properties are decisive when one wants to extract information from a lexical database efficiently or to update data while maintaining the coherence of the whole base.

The success of IG will also depend on their capacity to integrate other linguistic levels than the syntactic level, the scmantic level especially.

\section{References}

P. Blache. 1999. "Contraintes et théories linguistiques : des Grammaires d'Unification aux Grammaires de Propriétés". Thèse d'Habilitation, Université Paris 7.
M.-H Candito. 1999. Organisation modulaire et paramétrable de grammaires électroniques lexicalisées. Application au français et à l'italien,. Thèse de Doctorat, Université Paris 7.

D. Duchier and Gardent C. 1999. A constraintbased treatment of descriptions. In IWCS-3, Jan 99, Tillburg, The Netherlands.

D. Duchier and S. Thater. 1999. Parsing with tree descriptions: a constraint based approach. In NLULP'99,Dec 99, Las Cruces, New Mexico.

M. Egg, J. Niehren, P. Ruhrberg, and F. Xu. 1998. Constraints over lambda structires in semantic underspecification. In COLING/ACL'98, Aug 98, Montreal, Quebec, Canada.

J.-Y. Girard. 1987. Linear logic. Theoretical Computer Science, 50(1):1-102.

A. K. Joshi, L. S. Levy, and M. Takahashi. 1975. Tree adjunct grammars. Journal of Computer and System Sciences, 10(1):136-163.

L. Kallmeyer. 1999. Tree Description Grammars and Underspecified Representations. Ph.D. thesis, Universität Tübingen.

M. Kanovich. 1992. Horn programming in linear logic is NP-complete. In LICS'92, Jun 92, Santa Cruz, California, pages 200-210.

A. Kroch and A. Joshi. 1985. Linguistic relevance of tree adjoining grammars. Tochnical Report MSCI-85-18, Department of Computer and Information Science, University of Pemnsylvania.

J. Lambek. 1958. The mathematics of sentence structure. Amer. Math. Monthly, 65:154-169.

P. Lincoln. 1992. Computational aspects of linear logic. Ph.D. thesis, Stanford University.

M. Moortgart. 1996. Categorial Type Logics. In J. van Benthem and A. ter Meulon, editors, Handbook of Logic and Language, chaptier 2. Elsevier.

R. Muskens and E. Krahmer. 1998. Talking about trees and truth-conditions. In $L A C L^{\prime} 98$. Dec 98, Grenoble, France.

O. Rambow, K. Vijay-Shanker, and D. Weir. 1995. D-tree grammars. In ACL'95, pages 151-158.

C. Retoré. 2000. Systèmes déductifs et traitement des langues:un panorama des grammaires catégorielles. Research Report RR-3917, INRIA. To appear in Technique et Science Informatiques. Gert Smolka. 1995. The O't programming model. In Jan van Lceuwen, editor, Computer Science Today, Lecture Notes in Computer Science, vol. 1000, pages 324-343. Springer-Verlag, Berlin.

K. Vijay-Shanker. 1992. Using description of trees in a tree adjoining grammar. Computational Linguistics, 18(4):481-517. 